\title{
Modelling and solving a bi-objective intermodal transport problem of agricultural products
}

\author{
Abderrahman Abbassia ${ }^{*}$, Ahmed Elhilali Alaoui ${ }^{b}$ and Jaouad Boukachour ${ }^{c}$ \\ ${ }^{a}$ Ph.D Student, USMBA university, Faculty of Sciences and technology, Modeling and Scientific Calculus Laboratory, Fez, Morocco \\ ${ }^{b}$ Ph.D Professor, USMBA university, Faculty of Sciences and technology, Modeling and Scientific Calculus Laboratory, Fez, Morocco \\ ${ }^{c}$ Ph.D Professor, Normandie university, University of Le Havre, Applied Mathematics Laboratory, Le havre, France \\ CHRON I C L E \\ Article history: \\ Received August 182017 \\ Received in Revised Format \\ August 252017 \\ Accepted December 142017 \\ Available online \\ December 152017 \\ Keywords: \\ Intermodal transportation \\ Agricultural products export \\ Bi-objective optimization \\ NSGA-II \\ GRASP Algorithm \begin{abstract}
A B S T R A C T
During the past few years, transportation of agricultural products is increasingly becoming a crucial problem in supply chain logistics. In this paper, we present a new mathematical formulation and two solution approaches for an intermodal transportation problem. The proposed bi-objective model is applied to the transportation of agricultural products from Morocco to Europe to minimise both the transportation cost either in the form of uni-modal or intermodal, as well as the maximal overtime to delivery products. The first solution approach is based on a non-dominated sorting genetic algorithm improved by a local search heuristic and the second one is the GRASP algorithm (Greedy Randomised Adaptive Search Procedure) with iterated local search heuristics. They are tested on theoretical and real case benchmark instances and compared with the standard NSGA-II. Results are analysed and the efficiency of algorithms is discussed using some performance metrics.
\end{abstract}

Iterated local search

\section{Introduction}

Global consumption of agricultural products becomes much greater more than ever before because of demographic shifts of populations and the marketing progress of organic products. Morocco has given a serious priority to agriculture by developing several strategic projects such as the Green Morocco Plan to satisfy demands of its clients around the world with safe, sufficient, cheaper and high-quality products. According to some statistics of the agriculture ministry, there was a useful opportunity to make that sector to one of the pillars of the Moroccan economy with an average of $20 \%$ of the country's GDP and an important role in terms of jobs and activities especially in rural communities. Thanks to the important number of hectares allowed and the advanced regionalisation projects, all regions of the country contribute to produce millions of tons of vegetables and fruits. Some of the most convincing advantages of these projects are millions of workdays a year, billions of dirhams of profits and a remarkable abundance of different varieties of agricultural products, with significant commodities which are intended to customers worldwide. Because of the best geographical proximity of Moroccan ports and the quantity of products demanded, a considerable amount of goods of Moroccan farmers is heavily concentrated on the European continent which consumes an average of $90 \%$ of the exportation.

* Corresponding author

E-mail: abderrahman.abbassi@usmba.ac.ma (A. Abbassi)

2018 Growing Science Ltd.

doi: $10.5267 /$ j. ijiec.2017.12.001 
Previously the production was concentrated in the regions Souss-Massa-Drâa and Doukkala-Abda and it was massively intended for France. In recent years, most of the regions in Morocco produce and export to many high-potential markets of European countries. We focus our study on transportation of these goods from Moroccan suppliers to European customers to provide good planning for exporting their products.

We propose a new mathematical formulation totally different from the available models of intermodal transport problems, in order to minimise the cost and the overtime of transportation. The novelty of this work is its multi-objective version which is rarely addressed in intermodal transportation problems of the literature which are usually mono-objective problems. The overtime is the new objective function we propose in the mathematical model. Sometimes customers withdraw the order if the products arrive late. However, maybe it is illogical to cancel the order while the product is on the way and will be delivered after a reasonable delay. If the mode of transportation where the product is loaded needs a little additional time to arrive then why not accept it. This additional time is the overtime we look for minimizing. In addition, we take into account the flow management, time constraints and the lifetimes of products, which are not all taken into consideration simultaneously in the other works of the literature to the best of our knowledge. The new proposed model is solved using two proposed methods. But firstly, let us remind the principal steps of the exportation of agricultural products.

The agricultural supply chain involves the management of all activities, from harvesting to marketing (see Fig. 1). The harvest step has to be done quickly, with reduced damages and lower costs. Once products are harvested from fields, the preparation for markets consists in sorting and removing non-consumable elements, calibrating and packing products. To reduce costs of unnecessary tasks, it is recommended to sort products near the production fields. While washing, refrigeration and storage are needed to bring them to factory facilities that should benefit from an easy transportation access and a sufficient area to enable an easy movement and a fast loading and unloading of goods. The next step is loading products into containers where they will be ready for transportation. Using containers is necessary to protect products from friction, compression, temperature, humidity and contamination during transportation and to provide information about the product and its origin.

On the other hand, clients prefer to get the desired products at the desired time. They accept or refuse products based on the quality, appearance, freshness, colour and flavour. All these criteria are influenced by the transportation time, trip distance and the transport modes used. Once the products are ready, the transportation can be done from the production sites to customers using appropriate means of transport.

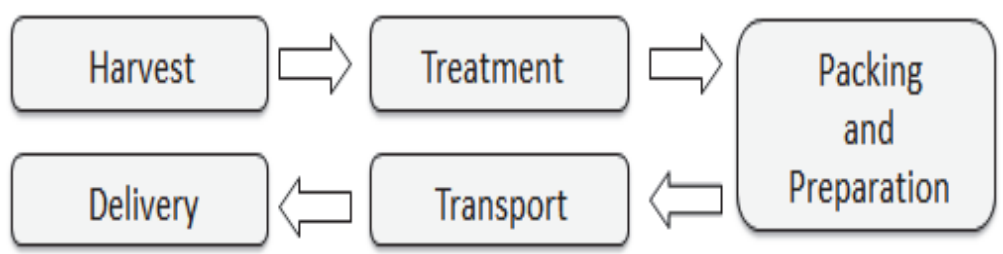

Fig. 1. Some steps of the agricultural supply chain

A significant part of the costs spent on managing the supply chain of agricultural products comes from transportation problems. In addition, transportation is almost the last step before delivering the product to customers. Even if the producers provide all operations with good decisions, if they do not give serious importance to the transportation step, they may ruin all efforts of the previous activities.

Generally, exportation of containers from Moroccan production sites to Europe can be ensured by 
road or ship transportation. The multimodality is reflected in the possibility of using two strategies of transportation. The first one is direct deliveries via road transportation. The second choice is intermodal shipment that combines two different modes of transportation, so transport units (containers) can be distributed from production sites in Morocco to suitable ports among potential terminals in the origin area (Port of Tangier-Med, for example), then to ports in the destination region, then finally to clients in Europe.

We can address two objectives: firstly, minimizing the total transportation cost of agricultural products, and secondly, minimizing the overtime objective. Indeed, these two objectives are contradictory. The cost of intermodal transportation is cheaper than direct shipment. However, its transportation time is higher; this is due to accumulated delays of transportation, services in terminals and a lot of intermediaries.

To solve this intermodal transportation problem, it is necessary to determine the optimal number of transportation units to be collected from each production site, the optimal terminals to use and the optimal routing of units through the network in order to optimize the objectives: the transportation cost and the overtime. Let us give a review of some works in the literature related to the problem of intermodal transport developed in this paper.

Researchers have always seen intermodal transport as an important problem in supply chain management and it has been widely studied in operation research. It can be defined as the transportation of goods or people from an origin area to a destination area using at least two different modes of transportation (Crainic \& Kim, 2007). Many articles in the literature have investigated intermodal transport problems. Arnold et al. (2004) developed a mathematical programme with mixed-integer variables to determine the optimal route along where the cargo will be transported and the best terminals to be used. Several extensions have developed that model. Sörensen et al. (2012) proposed two metaheuristics to solve the problem; the ABHC algorithm (Attribute Based Hill Climber) and GRASP (Greedy Randomised Adaptive Search Procedure) based on two phases of construction and improvement by a local search. The objective was to analyse the behaviour of each method for solving the intermodal terminal location problem. Results showed that there is no large difference in efficiency between the two solution approaches used by authors since the gap of deviation of the best solutions found from the exact solutions values was not very considerable. However, the parameter-free structure of the ABHC may make it in priority especially since the GRASP was not influenced by the increasing number of iterations according to their results. The same problem was again developed by Sörensen and Vanovermeire (2013) as a bi-objective model that minimises both the total cost function of intermodal transportation and the cost function of using terminals. They assume that the costs of the whole network of the intermodal terminal location problem are not paid by the same stakeholders. The hypothesis was to distinguish between the costs to be paid by the transporters of goods and the costs to be paid by the operators of the intermediate terminals. The model has also received the attention of Lin et al. (2014). They replaced two constraints by a simple and more efficient constraint which was proven to be equivalent. The new constraint purposes were to avoid redundancy in the model, improve the computational time and solve all benchmark instances.

All those works have addressed the problem in terms of intermodal terminal location. Other papers have tended to focus on the integration between production planning and intermodal transportation systems due to the global nature of the supply chain. In Meisel et al. (2013) authors combined production scheduling with intermodal transportation. They developed a mathematical programme to identify the optimum output of production sites, the amount of products incoming and outgoing from intermediate inventories at each period and the number of units to be transported using each mode. They looked for minimising the transportation cost and taking into consideration an environmental objective. The study was applied to a real case of chemical products in Europe. To solve the problem, an exact and a heuristic solution approach were adapted and the obtained results 
confirmed that intermodal transportation was the best choice from an economical point of view. In addition, there is no large impact of production scheduling on rail transportation, however transportation planning results in more shipment from production sites located near the destination area. In Chang (2008) a multi-objective optimization model was developed for minimizing transportation cost and time. They used a Lagrangian relaxation by integrating some constraints in the objective function using nonnegative penalty multipliers. They proposed a heuristic method that can be implemented easily after decomposing the master problem into a set of small subproblems. Moreover, Cho et al. (2012) studied a linear programming model of intermodal transportation for determining the shortest path from Busan to Rotterdam. In order to improve their solution approach, they used some heuristic called pruning rules for eliminating arcs with higher time and cost of transport.

Four years later, Lam and $\mathrm{Gu}$ (2016) developed a bi-objective mathematical model to minimize time and cost of container transit. Carbon dioxide (CO2) emissions are constrained and restricted by a deterministic value given by regulatory authorities; their results are applied to container export from China. Greenhouse emissions were also taken into account in the linear programming model proposed by Demir et al. (2016), but as an objective function associated with another function which minimizes transportation cost. In that study, they included demand and time uncertainties because these two factors have a crucial influence on the reliability of intermodal transport, according to the authors. Baykasoğlu and Subulan (2016) addressed a real-life case study of a logistics company in Turkey. The problem was mathematically formulated as a multi-objective model that contains three objective functions: the transportation cost through the network, the total transportation time, and the $\mathrm{CO} 2$ emissions by modes of transport. It aimed at managing the number of transportation units imported and exported during a multiperiod time horizon using three shipment modes (road-maritime-rail). The analysed results revealed that intermodal transportation was the efficient way for dispatching transportation units either in the economic point of view, the transit time or the sustainable objective.

Table 1

Some related papers of intermodal transport

\begin{tabular}{|c|c|c|c|c|c|c|c|c|c|c|c|c|c|}
\hline & \multicolumn{4}{|c|}{ Transport modes } & \multicolumn{4}{|c|}{ Objective functions } & \multicolumn{3}{|c|}{ Solution methods } & \multirow[b]{2}{*}{ 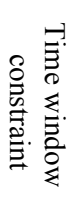 } & \multirow[b]{2}{*}{ 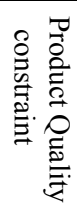 } \\
\hline & $\begin{array}{l}\widetilde{0} \\
\tilde{0} \\
\tilde{0}\end{array}$ & $\begin{array}{l}\underset{3}{2} \\
\text { : } \\
\text { : }\end{array}$ & $\begin{array}{l}\text { त्ठ․․ } \\
\text {. }\end{array}$ & $\stackrel{P}{B}$ & $\underset{\wp}{\approx}$ & $\vec{\Xi}$ & ᄋ & $\begin{array}{l}\frac{\pi}{\pi} . \\
\frac{\pi}{\pi}\end{array}$ & 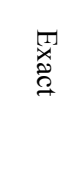 & 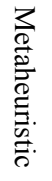 & 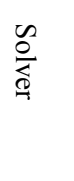 & & \\
\hline Choong et al. (2002) & $\sqrt{ }$ & $\sqrt{ }$ & $\sqrt{ }$ & & $\sqrt{ }$ & & & & & & $\sqrt{ }$ & & \\
\hline Arnold et al. (2004) & $\sqrt{ }$ & & $\sqrt{ }$ & & $\sqrt{ }$ & & & & & $\sqrt{ }$ & & & \\
\hline Erera et al. (2005) & $\sqrt{ }$ & $\sqrt{ }$ & $\sqrt{ }$ & & $\sqrt{ }$ & & & & & & $\sqrt{ }$ & $\sqrt{ }$ & \\
\hline Groothedde et al. (2005) & $\sqrt{ }$ & $\sqrt{ }$ & & & $\sqrt{ }$ & & & & & $\sqrt{ }$ & & & \\
\hline Chang (2008) & $\sqrt{ }$ & $\sqrt{ }$ & $\sqrt{ }$ & $\sqrt{ }$ & $\sqrt{ }$ & $\sqrt{ }$ & & & & $\sqrt{ }$ & & $\sqrt{ }$ & \\
\hline Limbourg and Jourquin (2009) & $\sqrt{ }$ & $\sqrt{ }$ & $\sqrt{ }$ & & $\sqrt{ }$ & & & & & $\sqrt{ }$ & & & \\
\hline Ishfaq and Sox (2010) & $\sqrt{ }$ & & $\sqrt{ }$ & $\sqrt{ }$ & $\sqrt{ }$ & & & & & $\sqrt{ }$ & & $\sqrt{ }$ & \\
\hline Sawadogo and Anciaux (2012) & $\sqrt{ }$ & $\sqrt{ }$ & $\sqrt{ }$ & $\sqrt{ }$ & $\sqrt{ }$ & $\sqrt{ }$ & $\sqrt{ }$ & $\sqrt{ }$ & & $\sqrt{ }$ & & & \\
\hline Cho et al. (2012) & $\sqrt{ }$ & $\sqrt{ }$ & $\sqrt{ }$ & $\sqrt{ }$ & $\sqrt{ }$ & $\sqrt{ }$ & & & & & & $\sqrt{ }$ & \\
\hline Meng and Wang (2011) & $\sqrt{ }$ & $\sqrt{ }$ & $\sqrt{ }$ & & $\sqrt{ }$ & & & & & $\sqrt{ }$ & & $\sqrt{ }$ & \\
\hline Sörensen et al. (2012) & $\sqrt{ }$ & & $\sqrt{ }$ & & $\sqrt{ }$ & & & & & $\sqrt{ }$ & & & \\
\hline Bierwirth et al. (2012) & $\sqrt{ }$ & & $\sqrt{ }$ & & $\sqrt{ }$ & & & & & & $\sqrt{ }$ & & \\
\hline Verma et al. (2012) & $\sqrt{ }$ & & $\sqrt{ }$ & & $\sqrt{ }$ & & & $\sqrt{ }$ & & $\sqrt{ }$ & & $\sqrt{ }$ & \\
\hline Alumur et al. (2012) & $\sqrt{ }$ & & & $\sqrt{ }$ & $\sqrt{ }$ & & & & & & $\sqrt{ }$ & $\sqrt{ }$ & \\
\hline Meisel et al. (2013) & $\sqrt{ }$ & & $\sqrt{ }$ & & $\sqrt{ }$ & & $\sqrt{ }$ & & $\sqrt{ }$ & $\sqrt{ }$ & & & \\
\hline Lin et al. (2014) & $\sqrt{ }$ & & $\sqrt{ }$ & & $\sqrt{ }$ & & & & & $\sqrt{ }$ & & & \\
\hline Etemadnia et al. (2015) & $\sqrt{ }$ & & & $\sqrt{ }$ & $\sqrt{ }$ & & & & & & $\sqrt{ }$ & & \\
\hline Lam and Gu (2016) & $\sqrt{ }$ & $\sqrt{ }$ & $\sqrt{ }$ & & $\sqrt{ }$ & $\sqrt{ }$ & & & & $\sqrt{ }$ & $\sqrt{ }$ & & \\
\hline This paper & $\sqrt{ }$ & $\sqrt{ }$ & & & $\sqrt{ }$ & $\sqrt{ }$ & & & & $\sqrt{ }$ & & $\sqrt{ }$ & $\sqrt{ }$ \\
\hline
\end{tabular}


Researches on intermodal transportation have not been limited to classical models; the strength of the problem of intermodal transport lies in the possibility of combining with other related problems. Several studies have combined it with real problems which can lead to this type of transport-specific characteristics of the case study addressed. In the last few years, many more applications of intermodal transport have become available, for instance: intermodal transportation of hazardous material (HAZMAT) in which we look for minimising transportation cost and the risk associated with the transportation of hazardous material (Jiang et al., 2014); wood transportation, freight activities of British intermodal rail (Woodburn, 2012); optimal links and locations for transportation hub in Marmara region (Resat \& Turkay, 2015); intermodal rail study from Asia to Europe (Rodemann \& Templar, 2014). The applications suggested in the fields of logistics are not exhaustive. In general, a recent survey of intermodal freight distribution can be found in Agamez-Arias and Moyano-Fuentes (2017) where a significant number of papers dealing with intermodal transport were reviewed and classified based on many factors.

In Table 1, we present some related papers by identifying modes of transportation used, the objective functions optimized, the implemented solution methods, the delivery time constraints and whether the product quality constraints were taken into account or not. As shown in Table 1, it is evident that road transportation remains the most competitive mode for intermodal freight transportation. Currently, it is characterized by good growth and its strength of competitiveness has reduced intermediaries and benefited from a worldly infrastructure which justifies the dominance of road transport in most articles. Intermodal transport contributes also significantly to the commodity. In our study we are interested in the road and maritime modes given the specificity of the transport networks Morocco-Europe.

In addition, maritime mode is large-scale; it is used to group a very significant amount of transport units. It is also characterized by low cost of transportation, which allows market access (De Mesquita \& Smith, 2009). Several mathematical models, academic or based on industrial applications, mainly investigated the minimization of the transportation cost and sometimes the transit time or an environmental objective. One of the main remarks in studies about intermodal transport is a lack of multi-objective problems. Minimizing the transportation cost represents an interest of transportation stakeholders. In our study we also considered an interest of clients by minimizing the needed supplementary time to receive products and taking into account the time constraints of deliveries. Such constraints are overlooked in many searches of the literature. The last column of Table 1 shows that researchers did not take into consideration the quality of the product which can be deteriorated during transport (we define these goods as perishable products).

Most papers are based only on the topology of intermodal transportation networks to give the best ways of using the network components (origin, ports, customers) and resources (modes). The questions that need to be also raised are: What will happen to the product during transportation? Will the paths and the modes chosen for transporting a product be necessarily the same for transporting another product? Maybe its lifetime requires another strategy of transport even if the transport network is the same, because to the contrary, the product will be delivered expired if the transportation time along the paths chosen exceeds its life time. Each product may require appropriate modes and paths to be delivered before its lifetime is expired. Hence, this constraint is very important and strong, it will be considered in our model by assuming that transportation time must not exceed the lifetime of the product transported.

The rest of this paper is organized as follows. The problem setting and the mathematical model for multi-objective intermodal transport of agricultural products are presented in section 2 . We propose the solution methods and we test them in section 3. The model and the approaches are applied again in section 4 to a case study of the Morocco-Europe network. Finally, section 5 provides a conclusion and future works. 


\section{Problem setting and mathematical model}

We consider an intermodal transport network that consists of four sets, namely a set $S$ of three production sites, a set $H$ of three terminals in the origin area, a set $T$ of four destination terminals and a set $C$ of four customers. That simple example is illustrated in Fig. 2.

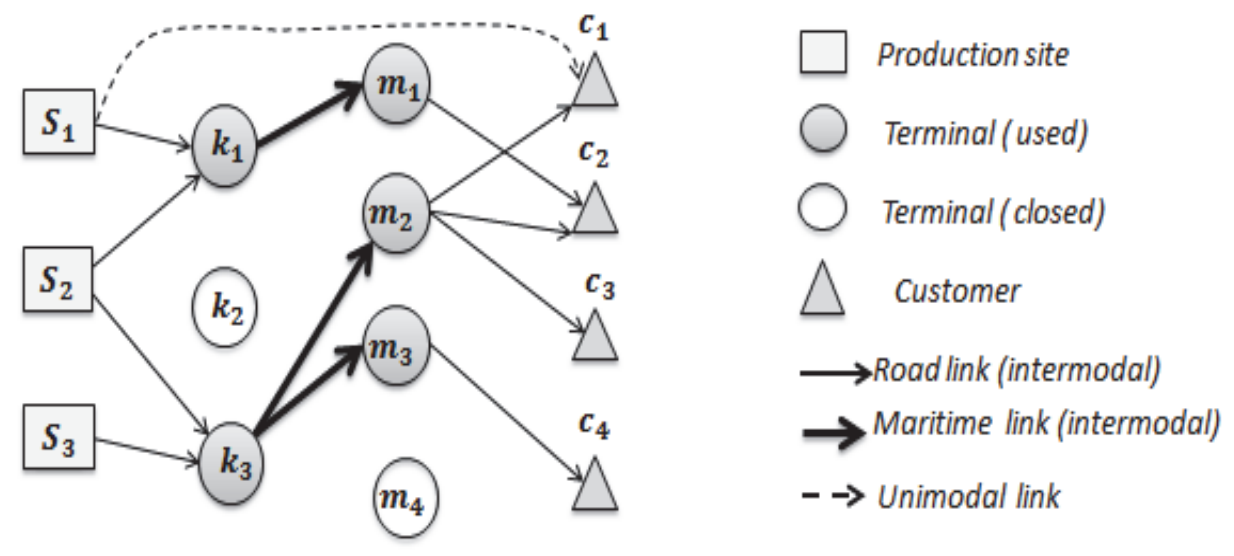

Fig. 2. Example of an intermodal transport network

As stated in the introduction, our aim was to optimise the exportation of agricultural products from the production sites. Each production site $i$ has a limited maximum capacity $\operatorname{Cap}_{i}$ measured in transportation units ( $1 \mathrm{TU}=1$ container). On the other side, each customer $j \in C$ has a demand $D_{e m}$ of the product measured as well in TU, and to be delivered taking into account the latest delivery time $L_{j}$. In other words, customer $j$ prefers to receive his demand before the latest arrival time $L_{j}$, otherwise the number of TUs requested will be refused. Sometimes, transport modes cannot arrive to customers before the latest date under any circumstances. So a supplementary time will be needed so that deliveries will be made. We denote "overtime" the supplementary time that the client needs so as to receive his total demands and we look for minimising it. In the network case described in Fig. 2, the shipper can use two types of transport services. Transport units can be sent by road transportation along a direct door-to-door shipment; such a service is represented by link $S_{1}-C_{1}$ in Fig. 2. They can also be shipped along intermodal links by road transport from sites $S$ to the terminals $H$ in the origin area, then by maritime mode to the terminals $T$ in the destination area, then finally by road modes to customers. Link $S_{2}-k_{3}-m_{3}-C_{4}$ represents such an intermodal transport path. In the first case, the carrier pays a transportation cost $\mathrm{c}_{\mathrm{ij}}$ for transporting one TU from a production site $i$ to the customer $j$. It also requires a transit time noted $t_{i j}$. While the second choice requires paying the transportation $\operatorname{cost} c_{i j}^{k m}$ from site $i$ to customer $j$ using terminal $k \in H$ and terminal $m \in$ $T$, without overlooking the travelling time $t_{i j}^{k m}$.

All used notations are summarized in Table 2, and except for the decision variables, all input data is deterministic and known in advance by the decision-maker. Note that costs are taken in euro (€) units, production capacities and demands of customers are both measured in transportation units, while the time of transportation is measured in hours.

\section{Table 2}

Notations used in the mathematical model

$\begin{array}{ll}S & \text { Set of production sites } \\ H & \text { Set of terminals in the origin area } \\ T & \text { Set of terminals in the destination area } \\ C & \text { Set of customers }\end{array}$


$c_{i j} \quad$ Cost of transport between site $\mathrm{i}$ and customer $\mathrm{j}$

$t_{i j} \quad$ The travel time between site $\mathrm{i}$ and customer $\mathrm{j}$

$\operatorname{Cap}_{i} \quad$ The capacity of production site $\mathrm{i}$

$\operatorname{Dem}_{j} \quad$ The demand of customer $\mathrm{j}$

$c_{i j}^{k m} \quad$ Transportation cost between site $\mathrm{i}$ and customer $\mathrm{j}$ using terminals $\mathrm{k}$ and $\mathrm{m}$

$t_{i j}^{k m} \quad$ Transportation time between site $\mathrm{i}$ and customer $\mathrm{j}$ using terminals $\mathrm{k}$ and $\mathrm{m}$

$T W_{j} \quad$ The latest time of product delivery for customer $\mathrm{j}$

$D W_{j} \quad$ The maximum delay allowed by the client $\mathrm{j}$

$T V \quad$ The lifetime of product

$w_{i j} \quad$ Binary decision variable that equals 1 if the product is transported directly from $\mathrm{i}$ to $\mathrm{j}$, and 0 otherwise

$x_{i j}^{k m} \quad$ Binary decision variable, it equals 1 if the product is sent from $\mathrm{i}$ to $\mathrm{j}$ using terminals $\mathrm{k}$ and $\mathrm{m}$, and 0 otherwise

$X_{i j} \quad$ Number of transportation units to be transmitted; it is the amount of product transported from the production site $\mathrm{i}$ to the customer $\mathrm{j}$

As mentioned previously, we aim to introduce a new bi-objective mathematical model for planning the intermodal transport of agricultural products. We recall that two necessary decisions have to be made: determining the optimal number of transportation units of the product to be transported from each production site to each customer, and the optimal itineraries to be used among direct or intermodal transportations. Using all necessary notations described in Table 2, the mathematical formulation is as follows:

$$
\min \sum_{\substack{i \in S \\ j \in C}} \varphi_{i j} \cdot X_{i j}
$$

$\min \max _{j \in C} O T_{j}$

$\sum_{j \in C} X_{i j} \leq \operatorname{Cap}_{i} \quad \forall i \in S$

$\sum_{i \in S} X_{i j}=\operatorname{Dem}_{j} \quad \forall j \in C$

$\sum_{\substack{k \in H \\ m \in T}} x_{i j}^{k m}+w_{i j} \leq 1 \quad \forall i \in S, \quad \forall j \in C$

$X_{i j} \leq M .\left(\sum_{\substack{k \in H \\ m \in T}} x_{i j}^{k m}+w_{i j}\right) \quad \forall i \in S, \quad \forall j \in C$

$\left(\sum_{\substack{k \in H \\ m \in T}} x_{i j}^{k m}+w_{i j}\right) \leq X_{i j} \quad \forall i \in S, \quad \forall j \in C$

$\sum_{\substack{k \in H \\ m \in T}} x_{i j}^{k m} \cdot t_{i j}^{k m}+w_{i j} \cdot t_{i j}-T W_{j} \leq D W_{j} \quad \forall i \in S, \quad \forall j \in C$

$\sum_{\substack{k \in H \\ m \in T}} x_{i j}^{k m} \cdot t_{i j}^{k m}+w_{i j} \cdot t_{i j} \leq T V \quad \forall i \in S, \quad \forall j \in C$

$X_{i j} \geq 0, x_{i j}^{k m} \in\{0,1\}, w_{i j} \in\{0,1\} \quad \forall i \in S, k \in H, m \in T, j \in C$ 
Constraints (3) bring into account the limited production capacity of each production site in the origin area. Constraints (4) ensure that the sum of all transportation units transported unimodally and intermodally from all production sites should equal the quantity of product requested by that customer $j$. Constraints (5) ensure the transport of goods only by the chosen links. If the product is directly transported from the site $i$ to the client $j$, then $w_{i j}=1$ and $x_{i j k m}=0$ for all terminals $k \in$ $H$ and for all terminals $m \in T$. If shipment is made via terminals $(k, m)$ then $w_{i j}=0$ and $x_{i j k m}=1$. Note that a production site is not obliged to give a product. In other words, the demand can be satisfied only by some production sites of the entire set $S$.

If shipment is made via an origin-destination link (either unimodal or intermodal), then the number of TUs to be sent must not exceed a greater value (we can just take $M=D e m_{j}$ ). Otherwise, there is nothing to carry through the unused links and the product has not left the production site; that is the aim of constraints (6). In the same manner, constraints (7) assume that links between any origindestination pair $(i, j)$ are not taken into consideration if there is no quantity of product to send to the customer $j$ by the production site $i$. Constraints (8) are for restricting the overtime. The supplementary time needed between any origin-destination pair should not exceed a limited value. That means that delays about the latest delivery time can be allowed, but they must not exceed the maximum allowed value $D W_{j}$.

As it is well known, agricultural products are fresh and in good quality once picked and their lifetimes are limited. The quality deteriorates and decreases over the time, especially for some products with a very short lifetime of 2 or 3 days and mostly if goods are transported in containers without a refrigeration system. As it is highlighted in Ramos et al. (2013), transportation time is one of the very influential factors on product quality and safety. Constraints (9) assume that transportation time of the product must not exceed its lifetime. The decision variables are defined in constraints (10) which ensure that only positive amounts can be transported, and each terminal should be used or not.

The first objective function (1) is minimizing the transportation cost of goods. The cost of transportation between a production site $i$ and a customer $j$ may be unimodal using a single mode, or intermodal using terminals and two modes. So it can be calculated as follows:

$$
\varphi_{i j}=\sum_{\substack{k \in H \\ m \in T}} c_{i j}^{k m} \cdot x_{i j}^{k m}+c_{i j} \cdot w_{i j} \quad \forall i \in S, \forall j \in C
$$

The second objective (2) is to minimize the maximal overtime for receiving total demands. We define the overtime associated to a customer $j$ as follows:

$$
O T_{j}=\max _{i \in S}\left(0, \sum_{\substack{k \in H \\ m \in T}} x_{i j}^{k m} \cdot t_{i j}^{k m}+w_{i j} \cdot t_{i j}-T W_{j}\right) \quad \forall j \in C
$$

Solving the bi-objective problem presented in this paper amounts to finding the very good approximate Pareto front which contains a set of non-dominated solutions. The comparison between the feasible elements of the research space is guaranteed thanks to the notion of Pareto optimality (in other words: the dominance between the solutions). Let $a$ and $b$ be two feasible solutions. We say that $a$ dominates $b$ if the value of $a$ is better than the value of $b$, i.e. if $f_{i}(a) \leq f_{i}(b) \forall i \in$ $\{1,2\}$ and $\exists j: f_{j}(a)<f_{j}(b)$. 
The problem studied in this paper is still new and original. There are no available instances for testing. So, before confirming our algorithms and applying them to solve real instances, we want firstly to test their efficiency on 14 randomly generated benchmarks, and we compare our results with those obtained by the standard non-dominated genetic algorithm. Firstly, we determine for each instance, the number of production sites, the number of origin terminals, the number of destination terminals and the number of customers in the network. Coordinates of each one of them are generated randomly in the Euclidian square between $(0,0)$ and $(2000,2000)$. The production site capacities and the demands of customers are respectively generated within the intervals $[0,300]$ and $[0,200]$, while lifetimes of products are taken as real data.

The unimodal transport cost between a production site $i$ and customer $j$ is equal to the Euclidian distance associated $d_{i j}\left(c_{i j}=d_{i j}\right)$. To allow discounts for using multimodal links, we determine their transport costs in a different manner. As in Lin et al. (2014), the cost of intermodal transport between the production site $i$ and customer $j$ through terminals $k$ and $m$ is $C_{i j k m}=C_{i k}+\frac{1}{2} C_{k m}+$ $C_{m j}$. Transportation time associated to each arc is equal to its distance. That means $t_{i j k m}=t_{i k}+$ $t_{k m}+t_{m j}$ and $t_{i j}=d_{i j}$. The latest arrival time $L_{j}$ of customer $j$ is randomly taken from the interval $\left[\mathrm{L}_{\text {minj }}, \mathrm{L}_{\text {maxj }}\right]$, where $L_{\text {minj }}$ represents the transportation time between customer $j$ and the nearest production site, while $L_{\max j}$ is the transport time between customer $j$ and the farthest production site. Details on the proposed methods will be given in the next section.

\section{Proposed solution approaches}

In order to solve the bi-objective mixed-integer model presented previously, we propose in this section two solution approaches, a hybrid non-dominated sorting genetic algorithm HNSGA and the GRASP algorithm with iterated local search. These methods require a simple coding for characterisation of solutions. We propose three parts for representation of an individual. The first one is an integer matrix $P X$ where the number of rows is the number of production sites and the number of columns equals the number of customers. This variable part describes the amount of product to be transported from each site to each customer. The second part $P x$ describing intermodal links is a matrix chromosome with four rows and the number of its columns equals the number of sites multiplied by the number of customers. The index of production sites is listed in the first line of this matrix, the index of customers in the fourth line, and the second and the third lines are respectively for origin and destination terminals between both sides. The representation of that part describing intermodal paths of Fig. 2 is shown in Fig. 3(A) where, as an explicative indication, the first column indicates that no terminals are used for transportation between the first production site and the first client. The third part $P w$ is another matrix with the same length of the $P X$ part but with binary values for representing unimodal links. The unimodal paths of Fig. 2 are represented by Fig. 3(B).

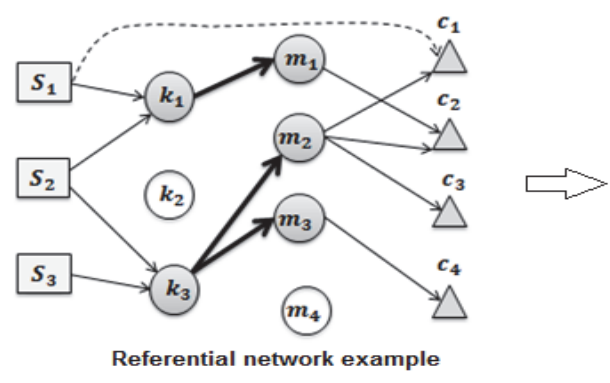

(A) : Px

\begin{tabular}{|l|l|l|l|l|l|l|l|l|l|l|l|}
\hline 1 & 1 & 1 & 1 & 2 & 2 & 2 & 2 & 3 & 3 & 3 & 3 \\
\hline 0 & 1 & 0 & 0 & 0 & 1 & 3 & 3 & 3 & 3 & 3 & 3 \\
\hline 0 & 1 & 0 & 0 & 0 & 1 & 2 & 3 & 2 & 2 & 2 & 3 \\
\hline 1 & 2 & 3 & 4 & 1 & 2 & 3 & 4 & 1 & 2 & 3 & 4 \\
\hline
\end{tabular}

(B) : Pw

\begin{tabular}{|l|l|l|l|}
\hline 1 & 0 & 0 & 0 \\
\hline 0 & 0 & 0 & 0 \\
\hline 0 & 0 & 0 & 0 \\
\hline
\end{tabular}

(C) : PX

\begin{tabular}{|c|c|c|c|}
\hline 20 & 50 & 0 & 0 \\
\hline 0 & 10 & 30 & 60 \\
\hline 0 & 10 & 70 & 10 \\
\hline
\end{tabular}

Fig. 3. Proposed representation of solutions 
Let us assume that each production site of the previous network example has a capacity of $100 \mathrm{TU}$ and the four clients of the same example have the following demands $\{20,70,100,70\}$ respectively. The third part $P X$ of an individual representing a solution for that example is shown in Fig. 3(C). In the following subsections, details of the proposed methods are given with parameter settings and performance analysis of each method according to some efficiency criteria.

\subsection{Hybrid non-dominated sorting genetic algorithm}

The NSGA-II is one of the well-known and widely used methods for solving problems with multiple objective functions. It consists in general in moving a population of $\mathrm{N}_{\text {ind }}$ individuals applying the operations of selection, crossover and mutation for diversification and looking for new feasible solutions by exploring the search space, until a stopping criterion is reached. At each iteration, solutions are grouped in different levels according to the dominance concept and step by step they will be updated until the last iteration when the best Pareto front is returned. Individuals are represented by the coding presented previously. Because an individual is represented by three parts $(P x, P w, P X)$, we apply three different operations of crossovers.

- The first one is applied to the $P x$ part of two different individuals by permuting two blocks. This can change the intermodal routes of the two individuals as shown in the following, where crossover of the parts $P x_{1}$ and $P x_{2}$ generates the new parts $E x_{1}$ and $E x_{2}$ :

\begin{tabular}{l|l|l|l|l|l|l|l|l|l|}
\hline \multirow{5}{*}{$P \times 1$} & 1 & 1 & 2 & 2 & 2 & 3 & 3 & 3 \\
\cline { 2 - 8 } & 2 & 1 & 1 & 2 & 2 & 2 & 2 & 3 & 3 \\
\hline 5 & 5 & 1 & 1 & 3 & 3 & 3 & 3 & 4 & 4 \\
\hline 1 & 2 & 3 & 1 & 2 & 3 & 1 & 2 & 3 \\
\hline
\end{tabular}

\begin{tabular}{|c|c|c|c|c|c|c|c|c|c|}
\hline \multirow{5}{*}{$E x 1$} & 1 & 1 & 2 & 2 & 2 & 3 & 3 & 3 \\
\hline \multirow{6}{*}{2} & 3 & 3 & 2 & 3 & 4 & 2 & 3 & 3 \\
\hline & 5 & 3 & 3 & 3 & 2 & 2 & 3 & 4 & 4 \\
\hline 1 & 2 & 3 & 1 & 2 & 3 & 1 & 2 & 3 \\
\hline
\end{tabular}

\begin{tabular}{|c|c|c|c|c|c|c|c|c|c|}
\hline \multirow{4}{*}{$P \times 2$} & 1 & 1 & 1 & 2 & 2 & 2 & 3 & 3 & 3 \\
\hline & 1 & 3 & 3 & 3 & 3 & 4 & 4 & 4 & 4 \\
\hline & 1 & 3 & 3 & 2 & 2 & 2 & 2 & 1 & 1 \\
\hline & 1 & 2 & 3 & 1 & 2 & 3 & 1 & 2 & 3 \\
\hline
\end{tabular}

\begin{tabular}{|c|c|c|c|c|c|c|c|c|c|}
\hline \multirow{4}{*}{$E \times 2$} & 1 & 1 & 1 & 2 & 2 & 2 & 3 & 3 & 3 \\
\hline & 1 & 1 & 1 & 3 & 2 & 2 & 4 & 4 & 4 \\
\hline & 1 & 1 & 1 & 2 & 3 & 3 & 2 & 1 & 1 \\
\hline & 1 & 2 & 3 & 1 & 2 & 3 & 1 & 2 & 3 \\
\hline
\end{tabular}

- The second step of crossover operation is applied to two $P w$ parts of two different individuals. It is done in a conventional manner by randomly selecting a cut point and exchanging the values of each block in order to change the unimodal routes of the two individuals as shown in the following:

\begin{tabular}{|c|c|c|c|c|c|c|c|c|c|}
\hline \multirow{4}{*}{$P w 1$} & 1 & 0 & 0 & 0 & \multirow{4}{*}{$E w 1$} & 1 & 0 & \begin{tabular}{|l|}
0 \\
\end{tabular} & 0 \\
\hline & 0 & 0 & 0 & 0 & & 0 & 0 & 0 & 0 \\
\hline & 0 & 1 & 1 & 1 & & 0 & 0 & 1 & 1 \\
\hline & 1 & 0 & 0 & 0 & & 1 & 1 & 0 & 0 \\
\hline & & & & & & & & & \\
\hline \multirow{4}{*}{ Pw2 } & 0 & 0 & 0 & 1 & \multirow{4}{*}{$E w 2$} & 0 & 0 & 0 & 1 \\
\hline & 0 & 0 & 0 & 0 & & 0 & 0 & 0 & 0 \\
\hline & 0 & 0 & 1 & 1 & & 0 & 1 & 1 & 1 \\
\hline & 0 & 1 & 0 & 0 & & 0 & 0 & 0 & 0 \\
\hline
\end{tabular}

- The third step of crossover requires a different manner by creating two children parts $\mathrm{EX}_{1}$ and $\mathrm{EX}_{2}$ from the barycentre of two parent parts $\mathrm{PX}_{1}$ and $\mathrm{PX}_{2}$ as follows: 


$$
E X_{1}=\alpha \cdot P X_{1}+(1-\alpha) \cdot P X_{2} \text { and } E X_{2}=(1-\alpha) \cdot P X_{1}+\alpha \cdot P X_{2}
$$

where $\alpha$ is randomly generated in $[0,1]$.

In order to explore the search space, it is also possible to apply the operation of mutation by modifying the genes of an individual to get other parts totally different. Using almost the same approach described previously in crossover step, the mutation of the $P x$ and the $P w$ parts of an individual is done by swapping some or all genes of the same individual. In other words, some positions are randomly chosen and their contents are exchanged as shown in the following example where the new part $E x$ is the result of mutation of $P x$.

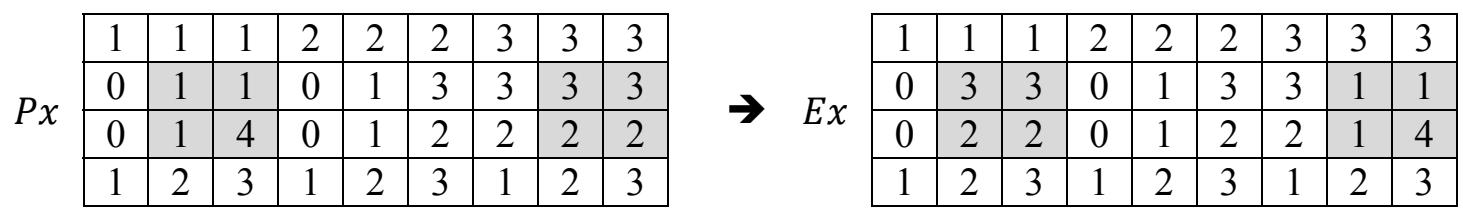

However, the mutation for an integer matrix $\mathrm{PX}_{1}$, for example, is done as follows:

$$
E X_{1}^{i}=\alpha \cdot P X_{1}^{i}+(1-\alpha) \cdot P X_{1}^{j} \text { and } E X_{1}^{j}=(1-\alpha) \cdot P X_{1}^{i}+\alpha \cdot P X_{1}^{j}
$$

where $\alpha$ is randomly generated in [0,1], $i$ and $j$ are two different positions of the integer part of the individual.

The major decision that needs to be made is to select terminals and routes to be used in the transport network and to determine the amount of the product that leaves each production site. For this reason and to improve our results, we use a local search which is one of the improving solution methods. It usually consists of two steps: building an initial solution and looking in the neighbourhood of the current solution for improving it in each iteration. The two-stage local search heuristic we used is applied after the NSGA-II approach. The first $H_{1}$ allows us to relocate other inactive terminals by choosing, for example, an open terminal that will be closed and replaced by another inactive terminal, as shown in Fig. 4 for terminals $k_{1}$ and $m_{3}$ which are replaced by $k_{2}$ and $m_{4}$. So, we separate the set of terminals of origin and destination areas into two subsets: $U$ which contains only the used terminals in the current solution found, and $F$ which contains the unused ones, then we apply a step of diversification and an intensification by changing the elements of the subsets as soon as possible and making sure that the new solutions are feasible.

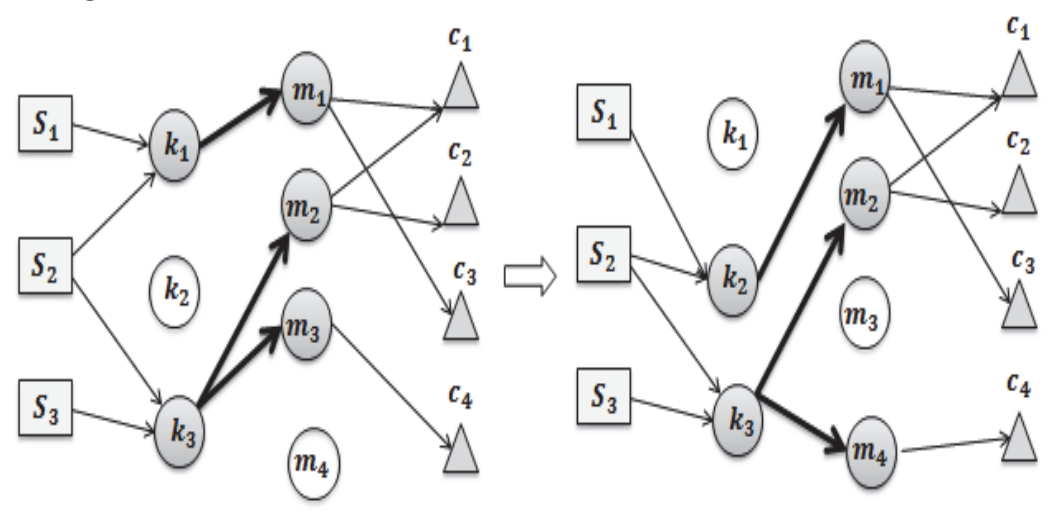

Fig. 4. Heuristic 1

The second heuristic $\mathrm{H}_{2}$ consists in changing routes using the same list of terminals already used, choosing two different production sites and exchanging their leaving flows of the product and their 
paths followed for arriving at the customer. This step is illustrated in Fig. 5 for sites $S_{1}$ and $S_{3}$.

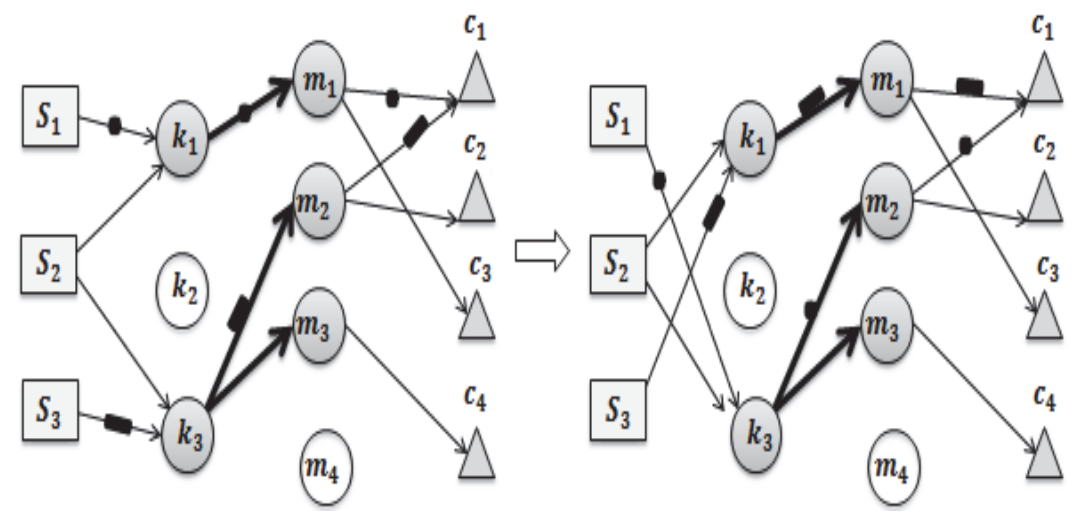

Fig. 5. Heuristic 2

In the worst case, if the solution found using these heuristics cannot be more improved, then we keep the solution found by the first phase (NSGA-II). Briefly, the main stages of this hybrid method proposed are shown in Algorithm 1.

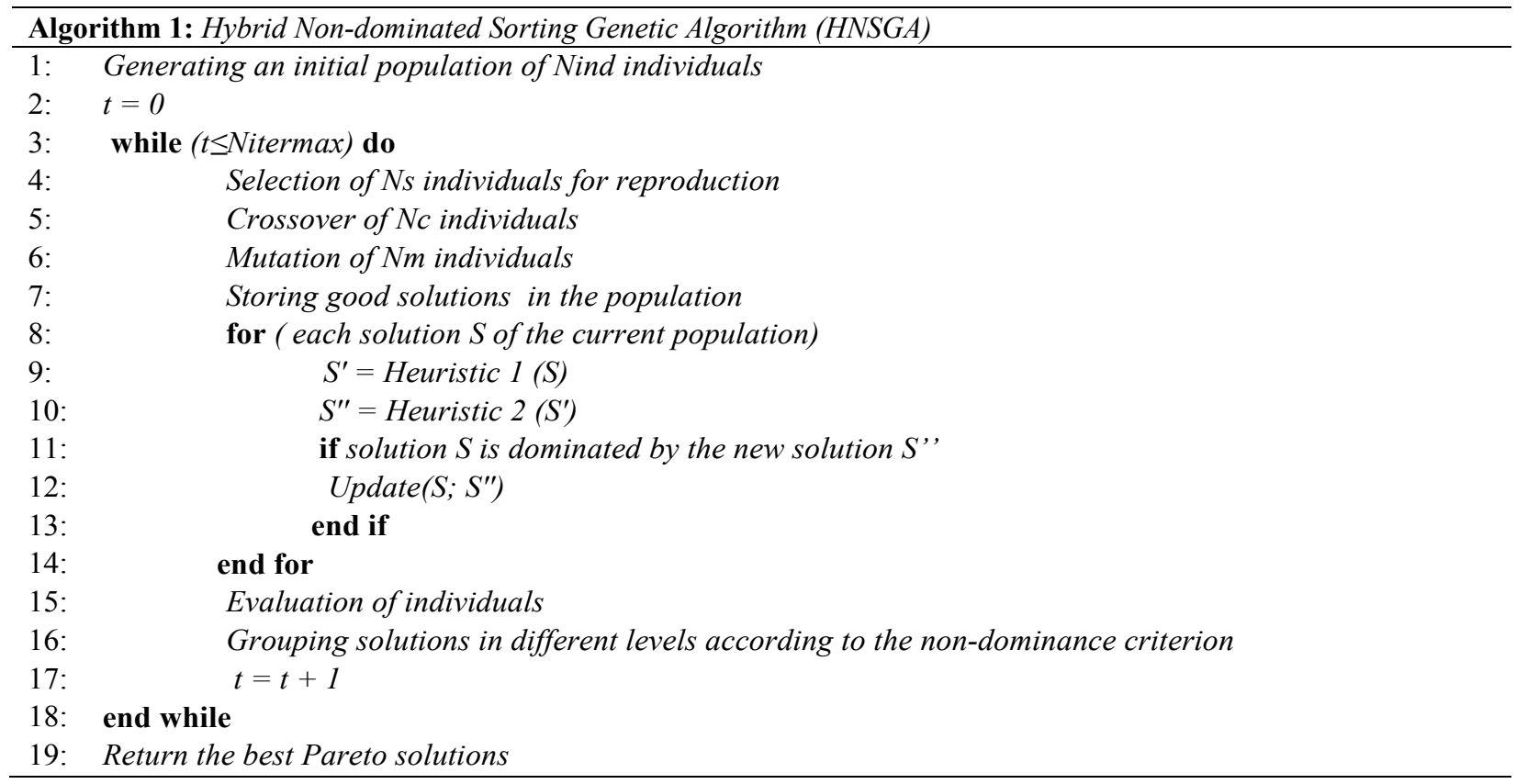

The second proposed method is described in the following subsection.

\subsection{Multi-objective GRASP algorithm with iterated local search (GRASP-ILS)}

The greedy randomised adaptive search procedure (GRASP) is a solution approach presented for the first time by Eberhart and Kennedy (1995). It is used for solving several combinatorial problems such as transportation (Ho \& Szeto, 2016), location problems (Hamidi et al., 2014), scheduling (Bierwirth \& Kuhpfahl, 2017), etc. The principle of that method is very simple: construction of an initial feasible solution then improving it at each iteration. We extend the application of this method in a bi-objective version for our problem and we hybridise it with an iterated local search procedure. The main steps of this method GRASP-ILS are presented in Algorithm 2 and described later. 


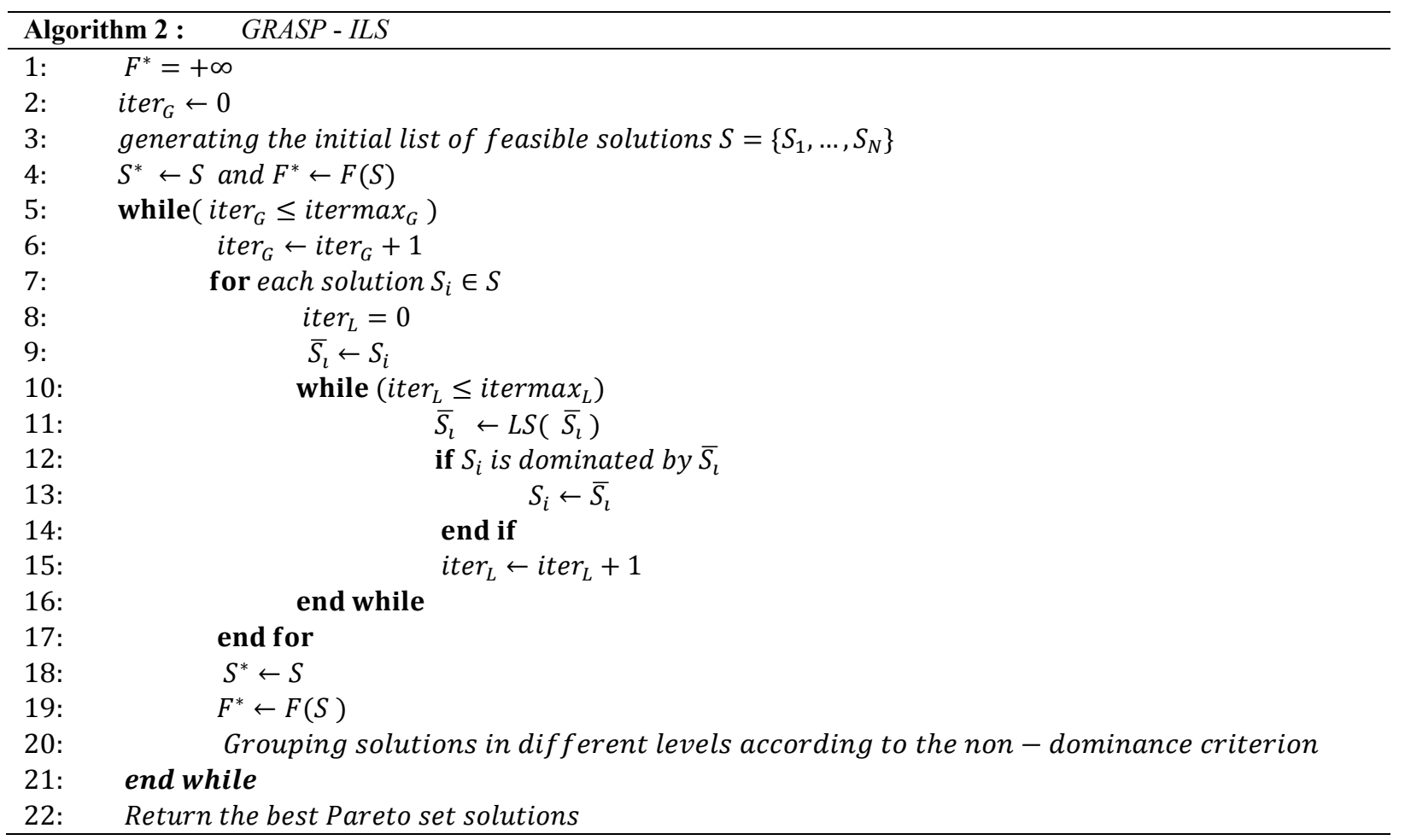

We generate an initial list of feasible solutions using a constructive procedure as in the initialisation step of the previous proposed method. The local search $L S$ used here for improving solutions is based on four heuristics:

- The first heuristic is Terminal-open which consists on choosing one or multiple closed terminals to replace other open terminals. It is the same heuristic $H_{1}$ described previously.

- The second heuristic is Path-swap for making an exchange of two paths between some sites and clients. Two executive terminals $(k, m)$ are randomly chosen and permuted with two other active terminals $\left(k^{\prime}, m^{\prime}\right)$ pertaining to another path as shown in Fig. 5 for terminals 11 and 3-2. This step is similar to the previous heuristic $H_{2}$.

- The third heuristic is Mode-swap. It consists in choosing a path between one production site and one customer then allowing transportation of goods directly using road transportation instead of the multimodal link and otherwise if goods are currently transported unimodally.

- The fourth heuristic is Integer local search. It consists in choosing one production site and two customers then revaluating their amounts. Assume that $X_{11}$ and $X_{31}$ are the amounts given to customers 1 by production sites 1 and 3 respectively. They will be updated as follows:

$$
X_{11}^{\text {new }} \leftarrow \operatorname{rand}\left(X_{11}+X_{31}\right) \quad \text { and } \quad X_{31}^{\text {new }} \leftarrow X_{11}+X_{31}-X_{11}^{\text {new }}
$$

This heuristic does not violate the satisfaction constraint of the customer's demands. However, it is necessary to ensure the satisfaction of capacity constraints of the production sites involved. 
In the updating step, if the neighbourhood found by local search heuristics is better, it replaces the local solution. Besides, the best local solution is also globally updated while a maximum number of iterations is not yet reached. Finally, the algorithm returns the best global non-dominated solutions found.

\subsection{Parameter settings and computational results}

The best choice of parameters has a considerable influence on result quality. For this reason, we launched the algorithms several times and with different values of crossover $\mathrm{Cr}$ and mutation $\mathrm{Mt}$ coefficients in order to identify the best parameters to be used instead of testing the methods for all instances with only one fixed parameter. Note that the stopping criterion is a maximum number of iterations fixed at 100 and the size of populations is fixed at 100 individuals. Values of the studied parameters influencing the behaviour of the proposed methods are shown in Table 3.

Table 3

Parameters setting

\begin{tabular}{lcccc}
\hline & & Value 1 & Value 2 & Value 3 \\
\hline Crossover coefficients & $\mathrm{Cr}$ & 0.3 & 0.6 & 0.8 \\
Mutation coefficients & $\mathrm{Mt}$ & 0.1 & 0.3 & 0.5 \\
\hline
\end{tabular}

The proposed methods are implemented in $\mathrm{C}++$ and run on a personal computer HP core i3, 2.2 $\mathrm{GHz}$ with $4 \mathrm{~GB}$ of RAM. Before testing these methods on the bi-objective problem, we want to use them for solving two mono-objective problems separately. The first one is with the cost function and the second is with the overtime function. This will allow us to find the ideal solutions for the bi-objective problem studied for each instance. We recall that a point $a$ is said to be the ideal point of a bi-objective problem if $a=\left(\min f_{1}, \min f_{2}\right)$. The best solutions of mono-objective problems are obtained with the values of parameters shown in Table 4.

Table 4

Values of parameters guaranteeing best solutions

\begin{tabular}{|c|c|c|c|c|c|c|c|c|c|c|c|c|}
\hline \multirow{3}{*}{ Instance } & \multicolumn{6}{|c|}{ cost } & \multicolumn{6}{|c|}{ overtime } \\
\hline & \multicolumn{2}{|c|}{ PM 1} & \multicolumn{2}{|c|}{ PM 2} & \multicolumn{2}{|c|}{ PM 3} & \multicolumn{2}{|c|}{ PM 1} & \multicolumn{2}{|c|}{ PM 2} & \multicolumn{2}{|c|}{ PM 3} \\
\hline & $\mathrm{Cr}$ & $\mathrm{Mt}$ & $\mathrm{Cr}$ & $\mathrm{Mt}$ & $\mathrm{Cr}$ & Mt & $\mathrm{Cr}$ & $\mathrm{Mt}$ & $\mathrm{Cr}$ & $\mathrm{Mt}$ & $\mathrm{Cr}$ & $\mathrm{Mt}$ \\
\hline 1 & 0.3 & 0.1 & 0.3 & 0.1 & - & - & 0.3 & 0.1 & 0.3 & 0.1 & - & - \\
\hline 2 & 0.3 & 0.5 & 0.7 & 0.5 & - & - & 0.3 & 0.5 & 0.3 & 0.3 & - & - \\
\hline 3 & 0.7 & 0.5 & 0.7 & 0.5 & - & - & 0.3 & 0.5 & 0.3 & 0.5 & - & - \\
\hline 4 & 0.7 & 0.5 & 0.5 & 0.3 & - & - & 0.5 & 0.1 & 0.3 & 0.5 & - & - \\
\hline 5 & 0.5 & 0.3 & 0.5 & 0.5 & - & - & 0.5 & 0.3 & 0.3 & 0.1 & - & - \\
\hline 6 & 0.3 & 0.5 & 0.3 & 0.5 & - & - & 0.5 & 0.3 & 0.3 & 0.1 & - & - \\
\hline 7 & 0.7 & 0.5 & 0.3 & 0.5 & - & - & 0.5 & 0.5 & 0.3 & 0.1 & - & - \\
\hline 8 & 0.5 & 0.5 & 0.5 & 0.3 & - & - & 0.3 & 0.1 & 0.3 & 0.1 & - & - \\
\hline 9 & 0.5 & 0.5 & 0.7 & 0.5 & - & - & 0.7 & 0.3 & 0.3 & 0.1 & - & - \\
\hline 10 & 0.3 & 0.3 & 0.3 & 0.1 & - & - & 0.3 & 0.5 & 0.3 & 0.1 & - & - \\
\hline 11 & 0.5 & 0.5 & 0.7 & 0.5 & - & - & 0.3 & 0.3 & 0.3 & 0.1 & - & - \\
\hline 12 & 0.7 & 0.5 & 0.7 & 0.5 & - & - & 0.7 & 0.5 & 0.3 & 0.1 & - & - \\
\hline 13 & 0.7 & 0.5 & 0.7 & 0.1 & - & - & 0.3 & 0.1 & 0.3 & 0.1 & - & - \\
\hline 14 & 0.7 & 0.5 & 0.7 & 0.5 & - & - & 0.5 & 0.3 & 0.3 & 0.1 & - & - \\
\hline
\end{tabular}

PM 1: NSGA-II , PM 2: HNSGA-II, PM 3: GRASP-ILS

In Table 5, the results obtained using the standard non-dominated sorting genetic algorithm NSGAII, the hybrid approach HNSGA-II and the values of solutions found by the GRASP algorithm with iterated local search refer respectively to the PM1, PM2 and PM3 columns. For each instance Inst, there is a specific number of sites $|S|$, a number $|H|$ of terminals in the origin area, $|T|$ destination 
terminals and $|C|$ customers. So, by analysing the mathematical model, we can easily calculate the numbers of constraints $N_{C}$ and decision variables $N_{D}$.

For the proposed method (GRASP-ILS) there are no crossover or mutation parameters to be fixed because these two operators are not used in this method. The best parameters for other methods are listed in the columns PM1 for the standard NSGA-II and PM2 for the hybrid HNSGA-II respectively. Even if the methods are the same algorithms used for solving all instances, however, each instance requires its appropriate values of parameters. In addition, the table shows that in the proposed method PM2, small values of crossover and mutation parameters are sufficient for obtaining very good results especially for solving the problem with the overtime function. The results obtained using those best parameters are shown in Table 5.

\section{Table 5}

Computational results obtained using the standard NSGA, the HNSGA approach and the GRASP with ILS for the mono-objective problems

\begin{tabular}{|c|c|c|c|c|c|c|c|c|c|c|c|c|}
\hline \multirow[b]{2}{*}{ Inst } & \multicolumn{4}{|c|}{$\begin{array}{c}\text { Network } \\
\text { characteristics }\end{array}$} & \multicolumn{2}{|c|}{$\begin{array}{c}\text { Model } \\
\text { characteristics }\end{array}$} & \multicolumn{3}{|c|}{ Cost } & \multicolumn{3}{|c|}{ Overtime } \\
\hline & $|\mathbf{S}|$ & $|\mathbf{H}|$ & $|\mathbf{T}|$ & $|\mathbf{C}|$ & $N_{D}$ & $N_{C}$ & PM 1 & PM2 & PM3 & PM 1 & PM2 & PM3 \\
\hline 1 & 5 & 5 & 5 & 5 & 675 & 185 & 208826 & 207541 & 193662 & 12 & 0 & 0 \\
\hline 2 & 10 & 10 & 10 & 10 & 10200 & 720 & 1201473 & 1116503 & 1268544 & 4 & 0 & 1 \\
\hline 3 & 20 & 10 & 10 & 10 & 20400 & 1330 & 950327 & 854629 & 919428 & 4 & 5 & 4 \\
\hline 4 & 30 & 10 & 10 & 10 & 30600 & 1940 & 1381143 & 1190567 & 1325595 & 5 & 1 & 1 \\
\hline 5 & 40 & 10 & 10 & 10 & 40800 & 2550 & 1327508 & 1004239 & 1112618 & 2 & 1 & 1 \\
\hline 6 & 10 & 20 & 10 & 10 & 20200 & 820 & 1002850 & 926558 & 983741 & 2 & 1 & 1 \\
\hline 7 & 10 & 30 & 10 & 10 & 30200 & 920 & 970727 & 763818 & 966114 & 2 & 0 & 0 \\
\hline 8 & 10 & 40 & 10 & 10 & 40200 & 1020 & 899671 & 693136 & 831549 & 0 & $\mathbf{0}$ & 0 \\
\hline 9 & 10 & 10 & 20 & 10 & 20200 & 820 & 1219668 & 1026389 & 1150416 & 4 & 2 & 2 \\
\hline 10 & 10 & 10 & 30 & 10 & 30200 & 920 & 890237 & 683089 & 817201 & 0 & 0 & 0 \\
\hline 11 & 10 & 10 & 40 & 10 & 40200 & 1020 & 986090 & 838804 & 962318 & 0 & 0 & 0 \\
\hline 12 & 10 & 10 & 10 & 20 & 20400 & 1330 & 2436929 & 2007116 & 2227321 & 6 & 4 & 6 \\
\hline 13 & 10 & 10 & 10 & 30 & 30600 & 1940 & 4514965 & 3888193 & 4121372 & 5 & 5 & 5 \\
\hline 14 & 10 & 10 & 10 & 40 & 40800 & 2550 & 5606904 & 4589579 & 4827404 & 11 & 9 & 9 \\
\hline
\end{tabular}

PM 1: NSGA-II , PM 2: HNSGA-II , PM 3: GRASP-ILS

In fact, for both mono-objective problems (problem with cost function and problem with overtime function), the GRASP-ILS method gives good solutions compared with the NSGA-II. However, the HNSGA-II is more efficient in comparison with other methods; it gives the best solutions nearly in all instances whatever their sizes. So in the mono-objective point of view, to minimise either the cost or the overtime, the hybrid approach HNSGA-II is still more efficient for solving the problem.

In the following, we analyse the efficiency of the proposed methods for solving the bi-objective problem and we will rely on some performance metric coefficients.

\subsection{Comparison metrics for bi-objective solutions}

As presented previously, we proposed two multi-objective algorithms and analysed their computational behaviour in addition to a comparison with the standard NSGA-II method. So as to analyse the quality of Pareto solutions obtained by those three methods, we use some popular performance metrics described as follows:

Mean ideal distance (MID): This indicates the mean distance between the ideal point and the Pareto solutions. The algorithm with lower value of median distance is more efficient. It can be computed by: 


$$
M I D=\sum_{i=1}^{n} \frac{c_{i}}{n}
$$

where $n$ is the number of non-dominated solutions and $c_{i}=\left\|f_{i}-f_{\text {ideal }}\right\|$.

Spread of non-dominated solutions (SNS): This is known as the diversity metric which measures the deviation of the ideal point from the non-dominated solutions, and the method with higher value has a better performance. This is calculated as follows:

$$
S N S=\sqrt{\frac{\sum_{i=1}^{n}\left(M I D-c_{i}\right)}{n-1}}
$$

Diversification metric $(\boldsymbol{D M})$ : This metric measures the diversity of Pareto solutions, and the algorithm with higher value for this metric brings better performance. It is calculated using the following equation:

$$
D M=\sqrt{\sum_{i=1}^{N}\left(\max f_{i}-\min f_{i}\right)^{2}}
$$

Domination percent (POD): This measures the efficiency of a solution approach in dominating other methods. All Pareto solutions found using all methods are mixed and grouped in one list so as to determine the global Pareto solutions set, then dominated solutions are eliminated. In this manner, we can calculate the percentage of non-dominated solutions found by each method.

The fourteen benchmark instances used for testing the proposed methods are solved for several times and the persuasive results are saved. Table 6 summarizes values of the principal performance metrics for each algorithm.

\begin{tabular}{|c|c|c|c|c|c|c|c|c|c|c|c|c|}
\hline \multirow[b]{2}{*}{ Instance } & \multicolumn{3}{|c|}{ MID $\left(\times 10^{3}\right)$} & \multicolumn{3}{|c|}{ SNS $\left(\times 10^{3}\right)$} & \multicolumn{3}{|c|}{ DM } & \multicolumn{3}{|c|}{ POD } \\
\hline & PM 1 & PM 2 & PM 3 & PM 1 & PM 2 & PM 3 & PM 1 & PM 2 & PM 3 & PM 1 & PM 2 & PM 3 \\
\hline 1 & 106.83 & 42.34 & 32.46 & 16.32 & 9.08 & 14.71 & 180.68 & 146.82 & 188.52 & 0 & 0 & 1 \\
\hline 2 & 388.67 & 213.04 & 310.13 & 61.42 & 89.49 & 114.12 & 425.11 & 551.14 & 508.16 & 0 & 0.8571 & 0.1429 \\
\hline 3 & 279.04 & 80.00 & 96.96 & 11.37 & 25.55 & 18.33 & 160.78 & 289.03 & 211.83 & 0 & 0.7778 & 0.2222 \\
\hline 4 & 409.89 & 127.19 & 242.12 & 27.23 & 15.33 & 123.10 & 233.38 & 225.34 & 518.27 & 0 & 0.8889 & 0.1111 \\
\hline 5 & 559.25 & 178.08 & 258.47 & 36.12 & 81.20 & 79.01 & 277.70 & 482.64 & 457.46 & 0 & 1 & 0 \\
\hline 6 & 193.55 & 44.93 & 21.07 & 3.04 & 5.89 & 0.00 & 81.89 & 146.54 & 0.00 & 0 & 0 & 1 \\
\hline 7 & 405.35 & 48.99 & 207.38 & 20.71 & 27.77 & 32.69 & 223.26 & 302.40 & 278.28 & 0 & 0.8333 & 0.1667 \\
\hline 8 & 352.11 & 27.74 & 97.71 & 0.00 & 6.87 & 0.00 & 0.00 & 120.90 & 0.00 & 0 & 1 & 0 \\
\hline 9 & 374.70 & 111.19 & 180.80 & 8.31 & 33.22 & 33.77 & 133.91 & 305.60 & 296.16 & 0 & 0.8889 & 0.1111 \\
\hline 10 & 321.50 & 19.37 & 167.65 & 2.99 & 1.23 & 17.11 & 77.49 & 52.84 & 226.24 & 0 & 1 & 0 \\
\hline 11 & 333.47 & 141.93 & 207.37 & 37.69 & 45.24 & 67.72 & 324.18 & 388.12 & 421.15 & 0 & 1 & 0 \\
\hline 12 & 809.58 & 187.24 & 368.87 & 87.40 & 67.22 & 174.20 & 482.11 & 454.82 & 675.36 & 0.2222 & 0.7778 & 0 \\
\hline 13 & 756.24 & 155.34 & 315.63 & 10.03 & 89.91 & 68.21 & 155.82 & 506.71 & 452.61 & 0 & 1 & 0 \\
\hline 14 & 1532.33 & 41.83 & 608.38 & 102.61 & 28.72 & 515.88 & 453.02 & 283.62 & 1139.40 & 0 & 0.7143 & 0.2857 \\
\hline
\end{tabular}

Table 6

Performance metrics for each algorithm

PM 1: NSGA-II, PM 2: HNSGA-II, PM 3: GRASP-ILS

Based on the mean ideal distance MID, the hybrid non-dominated sorting genetic algorithm HNSGA-II is more preferable than the GRASP with iterated local search and also than standard NSGA-II. 
The values of spread of non-dominated solutions SNS found using the hybrid HNSGA-II and the GRASP-ILS are higher than those found by the NSGA-II method which is not sufficiently efficient according to this criterion. However, the GRASP-ILS may be considered with a symbolic preference than the HNSGA-II since it ensures good SNS values in eight instances among the fourteen benchmarks. In addition, the standard NSGA-II indicates inferiority to the two proposed methods since its DM (Diversification metric) is with lower values for all instances. However, according to the DM values, there is no priority between the hybrid algorithm HNSGA-II and the GRASP-ILS and we cannot prefer one over another even if they are both better than the NSGA because of the number of instances where each one of those methods proves a superiority is nearly equivalent. Moreover, because of the higher values of domination percentage POD of solutions found using the HNSGA-II, this method is very preferable. A brief conclusion can be drawn according to the previous comparisons: for solving the problem studied, the NSGA-II method is the least efficient among the methods used, followed by the proposed GRASP algorithm with iterated local search which beckons for acceptable efficiency. However, the proposed hybrid method HNSGA-II is the most efficient and its results have a better performance according to the performance metric criteria.

In the next section we look more deeply into our study and we adapt our model and methods to a real case study.

\section{Case study}

In this section, we present an application of the bi-objective intermodal transport model, which we developed, on the Morocco-Europe network for planning the intermodal transportation of agricultural products of Moroccan farmers to some European cities. The real costs and times needed by the planning model are based on real distances between the real nodes of the network. Locations of origins, terminals and client nodes of the intermodal network are determined respectively based on the positions of regions where farmers produce and export the agricultural products, the positions of seaports through which commercial ships pass, and locations of some cities of the European continent. That data includes 37 nodes in total. There are 10 production sites chosen in the country, where agricultural products are available in sufficient amounts, 10 seaports which are considered as terminals in the origin area, 7 European seaports chosen as terminals in the destination area, and finally, 10 customers representing some cities of the European continent. Fig. 6 shows the locations and the corresponding nodes of the network for the studied case.

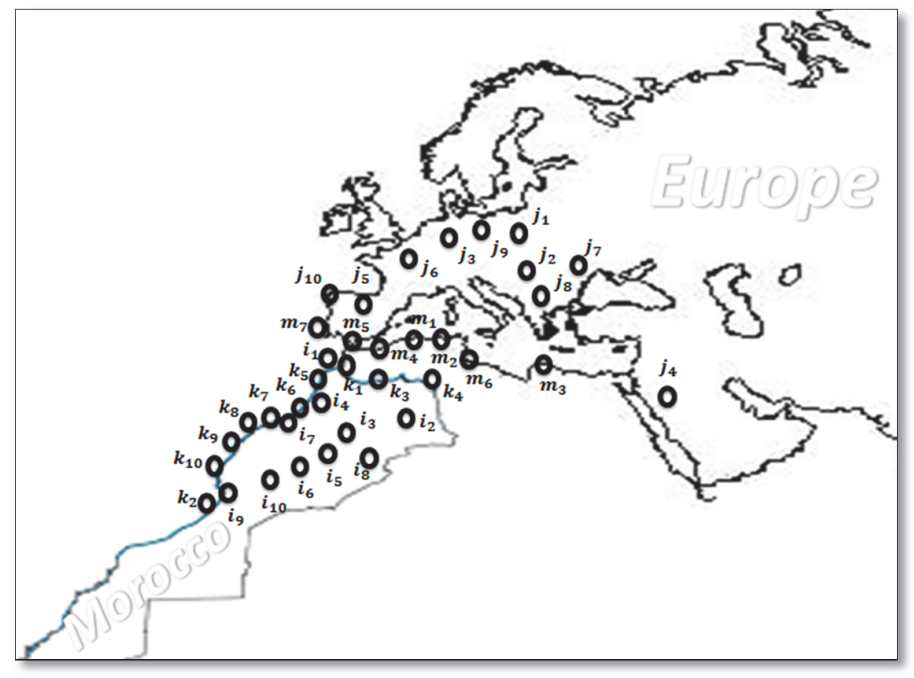

Fig. 6. Nodes of a real intermodal transport network 
The road transportation costs are calculated by multiplying the travelled distance by a unit cost $\mathrm{Cu}_{\mathrm{r}}=0.21(€)$ which is assumed to be the cost required for transportation by road mode of one transportation unit along one kilometre, while the maritime costs are taken to be equal to the travelled distance multiplied by a unit maritime transportation cost $\mathrm{Cu}_{\mathrm{m}}=0.17(€)$ which is assumed to be the cost for transporting by ship of one transportation unit along one mile. The unit transportation costs $\left(\mathrm{Cu}_{\mathrm{r}}\right.$ and $\left.\mathrm{Cu}_{\mathrm{m}}\right)$ are determined based on the proportionality between distances and costs in many real cases. Travelling times, using maritime transportation, are estimated by assuming that the merchant ships travel at $40 \mathrm{~km} / \mathrm{h}$ whereas transit times using road transportation are calculated assuming a travel speed of $80 \mathrm{~km} / \mathrm{h}$. Since we could not find any real values for demands, capacities and time windows, we complete our real instances by randomly generating them and choosing the latest arrival times of each customer between the lower and the higher values of travelled times needed for arriving at that customer. Table 7 shows the three categories of products used in real instances studied in this paper and their real lifetimes in days.

Table 7

Product lifetimes

\begin{tabular}{ccc}
\hline Category & Lifetime TV(days) & Product (Example) \\
\hline $\mathbf{1}$ & 2 & Strawberries \\
$\mathbf{2}$ & 4 & Courgettes \\
$\mathbf{3}$ & 10 & Apples \\
\hline
\end{tabular}

The first category is for products with short lifetime ( 2 days) such as strawberries. The second one is for a medium lifetime (4 days), for example courgettes, and the third one is for long lifetimes (10 days) such as apples. In each category, we tested four instances with different demands $\{20,50$, $100,500\}$. In the first instance of each category, each customer has a demand fixed at $20 \mathrm{TU}$. In the second one, the number of transport units demanded by each customer is fixed at $50 \mathrm{TU}$. In the third instance, it is fixed at $100 \mathrm{TU}$ and in the fourth instance, it is fixed at $500 \mathrm{TU}$. Which means, we tested 12 real benchmark instances.

In the following, we verify again the computational behaviour of each solution approach. Moreover, we study the effect of demand quantities on the choice of transportation modes and the choice of terminals to be used, in addition to the impact of lifetimes on the transportation strategy to be adopted.

Table 8 summarises the values of performance metrics for the three methods for each real problem. Briefly and similarly to the previous remarks, the proposed hybrid method HNSGA-II is very efficient and it indicates a superiority than other methods.

After solving these instances using the previous approaches, we analyze our obtained results (paths, flows). Even if we do not show to the honorable readers the paths obtained for all instances, for the sake of simplicity, we will just give a description of some best solutions obtained for three problems: they are the three instances with different lifetimes of products and the higher value of demands (as an example). Their solutions describe arcs and terminals of the best solutions obtained. However, all quantities transported will be presented as flow percentages in Fig. 7 which shows the average percentage of amounts that is transported through each seaport of the origin area or by the direct road transport (unimodal). 
Table 8

Performance metrics of the algorithms for the case study

\begin{tabular}{|c|c|c|c|c|c|c|c|c|c|c|c|c|}
\hline & \multicolumn{3}{|c|}{ MID $\left(\times 10^{3}\right)$} & \multicolumn{3}{|c|}{ SNS $\left(\times 10^{3}\right)$} & \multicolumn{3}{|c|}{ MD } & \multicolumn{3}{|c|}{ POD } \\
\hline & PM 1 & PM 2 & PM 3 & PM 1 & PM 2 & PM 3 & PM 1 & PM 2 & PM 3 & PM 1 & PM 2 & PM 3 \\
\hline Problem 1 & 91.43 & 11.58 & 36.79 & 76.09 & 9.74 & 21.45 & 390.10 & 161.01 & 207.13 & 0.1667 & 0.6667 & 0.1667 \\
\hline Problem 2 & 177.09 & 31.14 & 91.28 & 1.41 & 7.14 & 50.78 & 53.16 & 131.64 & 318.71 & 0 & 0.6 & 0.4 \\
\hline Problem 3 & 598.03 & $19 ., 64$ & 330.85 & 21.74 & 47.65 & 141.58 & 208.53 & 363.88 & 532.13 & 0 & 0.8 & 0.2 \\
\hline Problem 4 & 2421.73 & 766.84 & 1921.89 & 1609.19 & 266.76 & 789.83 & 1794.00 & 851.00 & 1333.00 & 0.1667 & 0.6667 & 0.1667 \\
\hline Problem 5 & 85.06 & 12.21 & 31.72 & 2.57 & 8.82 & 6.39 & 112.88 & 174.40 & 123.98 & 0 & 1 & 0 \\
\hline Problem 6 & 182.99 & 34.65 & 67.90 & 30.04 & 24.32 & 37.58 & 402.02 & 292.22 & 284.78 & 0.125 & 0.75 & 0.125 \\
\hline Problem 7 & 367.32 & 55.84 & 153.68 & 12.40 & 46.15 & 44.19 & 157.52 & 399.14 & 351.00 & 0 & 1 & 0 \\
\hline Problem 8 & 1950.37 & 336.86 & 925.55 & 178.75 & 344.70 & 245.95 & 768.56 & 912.24 & 815.86 & 0 & 1 & 0 \\
\hline Problem 9 & 129.43 & 24.51 & 32.85 & 10.26 & 13.86 & 9.44 & 143.27 & 177.18 & 161.97 & 0 & 0.6 & 0.4 \\
\hline Problem 10 & 235.80 & 30.41 & 77.85 & 3.64 & 27.46 & 30.37 & 85.33 & 267.19 & 284.36 & 0 & 1 & 0 \\
\hline Problem 11 & 503.75 & 59.91 & 112.98 & 36.17 & 57.40 & 67.94 & 294.27 & 397.00 & 380.64 & 0 & 1 & 0 \\
\hline Problem 12 & 2552,17 & 334.02 & 845.70 & 446.94 & 305.77 & 253.60 & 1678.70 & 923.10 & 829.02 & 0.125 & 0.75 & 0.125 \\
\hline
\end{tabular}

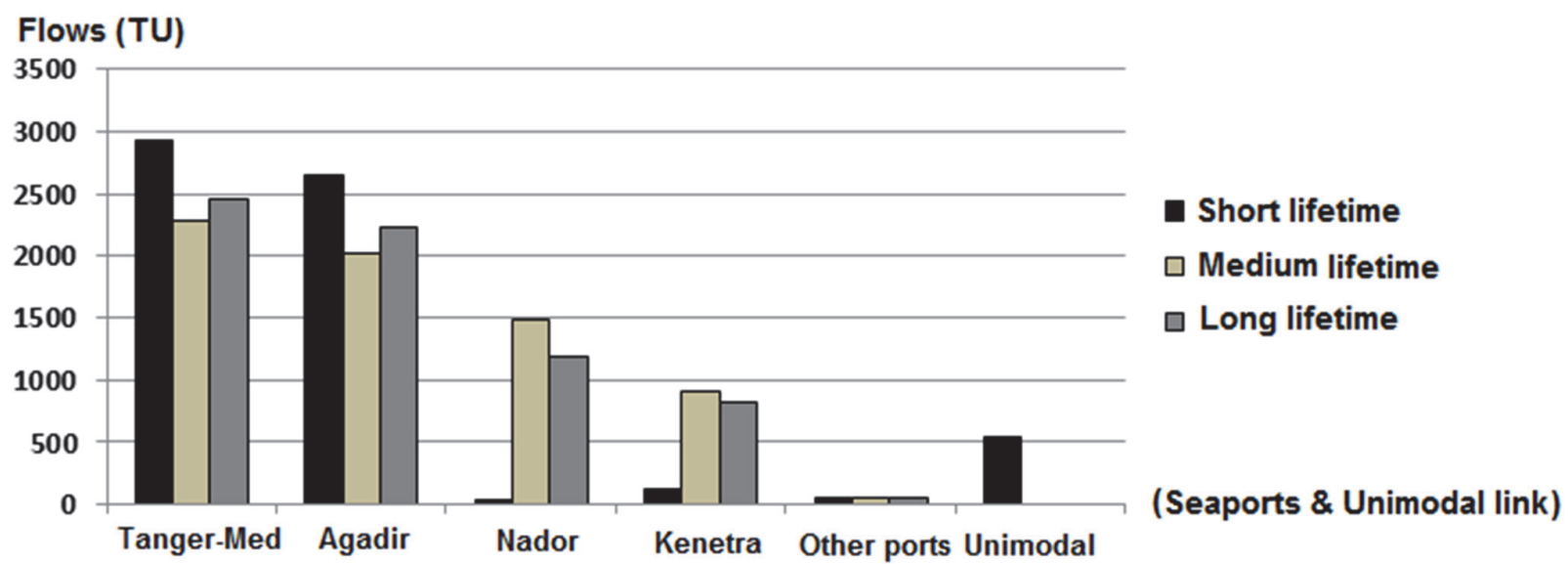

Fig. 7. Percentage of goods transported through seaports of the origin area

These results revealed that the seaports of the studied network do not have the same importance and each product may require a specific list of terminals and modes for assuring its transportation. Moreover, for all products whatever their lifetimes, there is a dominance of combined transportation (road and maritime modes) than direct road transportation, and the higher amount of goods has to be exported through the four seaports located in the north and the north-east of Morocco: Tangiermed, Agadir, Nador and Kenitra. Let us call these seaports the best terminals. While other seaports have to be unused. For products with medium and long lifetimes, the direct road link (unimodal) is not recommended for exporting these categories of products because there is no flow sent along unimodal paths. However, for transporting products with short lifetime, the ports of Tangier-Med and Agadir are the most appropriate terminals to be used and some customers have to be also served using direct road mode (unimodal); maybe its speed has an advantage for this category of products. Solutions indicating paths to be followed for transporting products are shown in Fig 8 .

As a brief conclusion, many Moroccan farmers used to export their products via seaports of Tangier or Agadir whatever their transported product, whatever its lifetime and whatever the amount of product transported. However, the analyzed results showed that it is not enough to generally determine the paths to be followed during transportation, but the choice of modes of transportation, terminals, and the amounts of goods, depend on the product transported itself and its lifetime. In other words, each product may have its own paths and suitable modes for transporting it to customers with minimum cost and minimum supplementary time. 


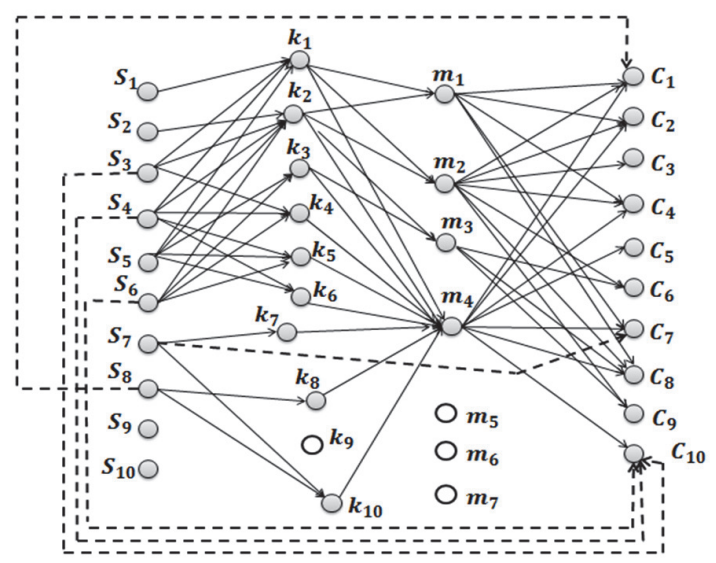

(A) Short lifetime

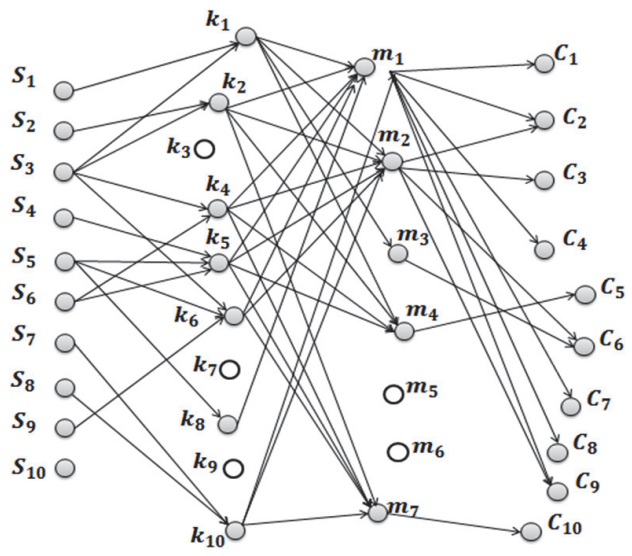

(B) Medium lifetime

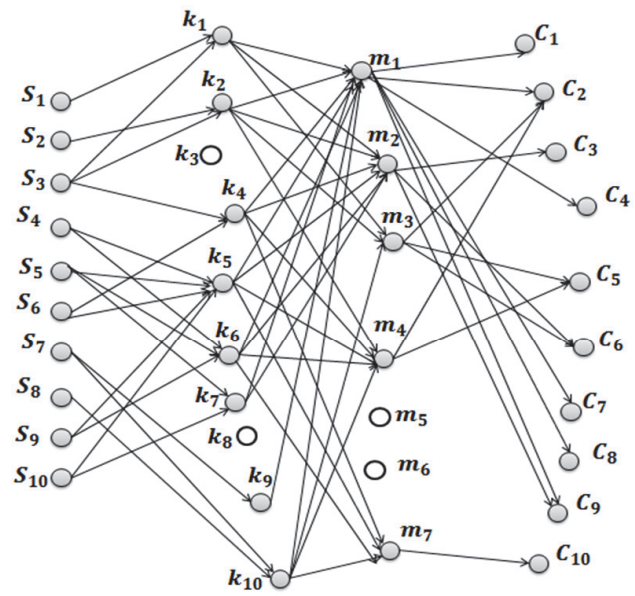

(C) Long lifetime

Fig. 8. Some best solutions obtained for three products categories with different lifetimes

\section{Conclusion}

In this paper, we introduced a new mathematical model and we developed two hybrid solution approaches for the intermodal transport of agricultural products from Morocco to Europe; it is a new contribution to the literature in intermodal transport network problems. The model includes various observations from real life and it is applied to agricultural product exportation using direct road mode or intermodal transportation which combines maritime and road modes. This study is also intended to be applicable to intermodal transport of any perishable product which has a lifetime taking into consideration keeping quality of goods before reaching the customers, because not only the network typology has to be taken into consideration, but also transported product characteristics have to be taken into account in the transport strategy. Indeed, the problem we proposed is a biobjective combinatorial optimisation problem, nonlinear and mixed. It is harder to be solved with exact algorithms or solvers (as Cplex). Thus, we adapted meta-heuristic methods for solving that problem. The first method we proposed is the hybrid non-dominated sorting genetic algorithm HNSGA-II. The second proposed approach is the GRASP method with iterated local search, GRASP-ILS, and both methods are also compared with the standard NSGA-II. Theoretical analysis is done so as to study the efficiency of the proposed algorithms which are tested on randomly generated instances; they are used more efficiently by setting the best parameters of the solution approaches. In mono- and bi-objective points of view, the hybrid method HNSGA-II is more 
efficient than the GRASP algorithm with iterated local search and the standard NSGA-II according to the obtained results and the performance metrics values. Real application is also done on a real intermodal transport network, Morocco-Europe, where the hybrid method HNSGA-II proves again its efficiency. That allows us to draw several remarks about the most suitable and useful terminals and to propose some recommendations that should be considered by decision-makers. Many suggestions might be the subject of our future researches. For example, extending to cope with uncertain demands of customers. Moreover, last mile management is a very important problem which has to be highlighted in such study for serving customers residing in dense or urban areas. Time and cost of distribution in that case could change the whole strategy and the network design.

\section{References}

Agamez-Arias, A. D. M., \& Moyano-Fuentes, J. (2017). Intermodal transport in freight distribution: a literature review. Transport Reviews, 1-26.

Alumur, S. A., Kara, B. Y., \& Karasan, O. E. (2012). Multimodal hub location and hub network design. Omega, 40(6), 927-939.

Arnold, P., Peeters, D., \& Thomas, I. (2004). Modelling a rail/road intermodal transportation system. Transportation Research Part E: Logistics and Transportation Review, 40(3), 255-270.

Baykasoğlu, A., \& Subulan, K. (2016). A multi-objective sustainable load planning model for intermodal transportation networks with a real-life application. Transportation Research Part E: Logistics and Transportation Review, 95, 207-247.

Bierwirth, C., \& Kuhpfahl, J. (2017). Extended GRASP for the job shop scheduling problem with total weighted tardiness objective. European Journal of Operational Research, 261(3), 835-848.

Bierwirth, C., Kirschstein, T., \& Meisel, F. (2012). On transport service selection in intermodal rail/road distribution networks.

Chang, T. S. (2008). Best routes selection in international intermodal networks. Computers \& operations research, 35(9), 2877-2891.

Cho, J. H., Kim, H. S., \& Choi, H. R. (2012). An intermodal transport network planning algorithm using dynamic programming - a case study: from Busan to Rotterdam in intermodal freight routing. Applied Intelligence, 36(3), 529-541.

Choong, S. T., Cole, M. H., \& Kutanoglu, E. (2002). Empty container management for intermodal transportation networks. Transportation Research Part E: Logistics and Transportation Review, $38(6), 423-438$.

Crainic, T. G., \& Kim, K. H. (2007). Intermodal transportation. Handbooks in operations research and management science, 14, 467-537.

De Mesquita, B. B., \& Smith, A. (2009). A political economy of aid. International Organization, 63(2), 309-340.

Demir, E., Burgholzer, W., Hrušovský, M., Arıkan, E., Jammernegg, W., \& Van Woensel, T. (2016). A green intermodal service network design problem with travel time uncertainty. Transportation Research Part B: Methodological, 93, 789-807.

Eberhart, R., \& Kennedy, J. (1995, October). A new optimizer using particle swarm theory. In Micro Machine and Human Science, 1995. MHS'95., Proceedings of the Sixth International Symposium on (pp. 39-43). IEEE.

Erera, A. L., Morales, J. C., \& Savelsbergh, M. (2005). Global intermodal tank container management for the chemical industry. Transportation Research Part E: Logistics and Transportation Review, 41(6), 551-566.

Etemadnia, H., Goetz, S. J., Canning, P., \& Tavallali, M. S. (2015). Optimal wholesale facilities location within the fruit and vegetables supply chain with bimodal transportation options: An LP-MIP heuristic approach. European Journal of Operational Research, 244(2), 648-661.

Groothedde, B., Ruijgrok, C., \& Tavasszy, L. (2005). Towards collaborative, intermodal hub networks: A case study in the fast moving consumer goods market. Transportation Research Part E: Logistics 
and Transportation Review, 41(6), 567-583.

Hamidi, M., Farahmand, K., Sajjadi, S., \& Nygard, K. (2014). A heuristic algorithm for a multi-product four-layer capacitated location-routing problem. International Journal of Industrial Engineering Computations, 5(1), 87-100.

Ho, S. C., \& Szeto, W. Y. (2016). GRASP with path relinking for the selective pickup and delivery problem. Expert Systems with Applications, 51, 14-25.

Ishfaq, R., \& Sox, C. R. (2010). Intermodal logistics: The interplay of financial, operational and service issues. Transportation Research Part E: Logistics and Transportation Review, 46(6), 926-949.

Jiang, Y., Zhang, X., Rong, Y., \& Zhang, Z. (2014). A multimodal location and routing model for hazardous materials transportation based on multi-commodity flow model. Procedia-Social and Behavioral Sciences, 138, 791-799.

Lam, J. S. L., \& Gu, Y. (2016). A market-oriented approach for intermodal network optimisation meeting cost, time and environmental requirements. International Journal of Production Economics, 171, 266274.

Limbourg, S., \& Jourquin, B. (2009). Optimal rail-road container terminal locations on the European network. Transportation Research Part E: Logistics and Transportation Review, 45(4), 551-563.

Lin, C. C., Chiang, Y. I., \& Lin, S. W. (2014). Efficient model and heuristic for the intermodal terminal location problem. Computers \& Operations Research, 51, 41-51.

Meisel, F., Kirschstein, T., \& Bierwirth, C. (2013). Integrated production and intermodal transportation planning in large scale production-distribution-networks. Transportation Research Part E: Logistics and Transportation Review, 60, 62-78.

Meng, Q., \& Wang, X. (2011). Intermodal hub-and-spoke network design: incorporating multiple stakeholders and multi-type containers. Transportation research Part B: Methodological, 45(4), 724742.

Ramos, B., Miller, F. A., Brandão, T. R., Teixeira, P., \& Silva, C. L. (2013). Fresh fruits and vegetablesan overview on applied methodologies to improve its quality and safety. Innovative Food Science \& Emerging Technologies, 20, 1-15.

Resat, H. G., \& Turkay, M. (2015). Design and operation of intermodal transportation network in the Marmara region of Turkey. Transportation Research Part E: Logistics and Transportation Review, 83, 16-33.

Rodemann, H., \& Templar, S. (2014). The enablers and inhibitors of intermodal rail freight between Asia and Europe. Journal of Rail Transport Planning \& Management, 4(3), 70-86.

Sawadogo, M., \& Anciaux, D. (2012). Sustainable supply chain by intermodal itinerary planning: a multiobjective ant colony approach. International Journal of Agile Systems and Management, 5(3), 235-266.

Sörensen, K., \& Vanovermeire, C. (2013). Bi-objective optimization of the intermodal terminal location problem as a policy-support tool. Computers in Industry, 64(2), 128-135.

Sörensen, K., Vanovermeire, C., \& Busschaert, S. (2012). Efficient metaheuristics to solve the intermodal terminal location problem. Computers \& Operations Research, 39(9), 2079-2090.

Verma, M., Verter, V., \& Zufferey, N. (2012). A bi-objective model for planning and managing railtruck intermodal transportation of hazardous materials. Transportation research part E: logistics and transportation review, 48(1), 132-149.

Woodburn, A. (2012). Intermodal rail freight activity in Britain: Where has the growth come from?. Research in Transportation Business \& Management, 5, 16-26.

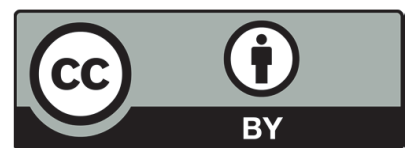

C 2018 by the authors; licensee Growing Science, Canada. This is an open access article distributed under the terms and conditions of the Creative Commons Attribution (CCBY) license (http://creativecommons.org/licenses/by/4.0/). 